\title{
„Ah Dieu! que la guerre est jolie“ (Apollinaire). Die ästhetische Valorisierung des Kriegs durch die französische Avantgarde
}

"Ah Dieu! que la guerre est jolie» (Apollinaire). La valorisation esthétique de la guerre par l'avant-garde en France

"Ah Dieu! que la gerre est jolie" (Apollinaire). The aesthetic valorisation of the war through the avant-garde in France

Joseph Jurt

\section{OpenEdition}

\section{Journals}

Édition électronique

URL : http://journals.openedition.org/ceg/1999

DOI : $10.4000 /$ ceg. 1999

ISSN : 2605-8359

Éditeur

Presses Universitaires de Provence

Édition imprimée

Date de publication : 15 juin 2014

Pagination : 105-116

ISSN : 0751-4239

\section{Référence électronique}

Joseph Jurt, « „Ah Dieu ! que la guerre est jolie“ (Apollinaire). Die ästhetische Valorisierung des Kriegs durch die französische Avantgarde », Cahiers d'Études Germaniques [Online], 66 | 2014, Online erschienen am: 17 Dezember 2017, abgerufen am 18 November 2020. URL : http:// journals.openedition.org/ceg/1999; DOl : https://doi.org/10.4000/ceg.1999 


\section{«Ah Dieu ! que la guerre est jolie » (Apollinaire). Die ästhetische Valorisierung des Kriegs durch die französische Avantgarde}

Joseph JURT

Universität Freiburg

1989 veröffentlichte der amerikanische Kunsthistoriker Kenneth E. Silver eine Monographie zur Pariser Avantgarde und dem Ersten Weltkrieg, die schon 1991 auf Französisch unter dem Titel Vers le retour à l'ordre. L'avantgarde parisienne et la première guerre mondiale erschien. ${ }^{1}$ Nach Kenneth E. Silver bedeutete die 'Union sacrée' de facto eine Quasi-Kapitulation der Linken vor der Rechten. Jene mussten nun beweisen, dass sie auch Patrioten seien, selbst die avantgardistische Kunst, die sich vor dem Krieg durch ihre internationale Dimension ausgezeichnet hatte, stellte nun patriotische Werte in den Mittelpunkt. Das kulturelle Paradigma der Rechten, wie es die Action française artikuliert hatte (die Verbindung von Klassizismus und Nationalismus), wurde zur Dominante. Ein dekadentes, verrücktes, kosmopolitisches Vorkriegsfrankreich sei durch den Krieg zur Vernunft gebracht worden. ${ }^{2}$

Ich bin mir nicht sicher, ob man von einer solchen generellen Wende zum Paradigma der Rechten sprechen kann. Zweifellos teilten nun beide Seiten einen patriotischen Konsens auf der Basis dessen, was Poincaré in seiner 'Union sacrée'-Rede vom August 1914 formuliert hatte (,In dem Krieg, der jetzt entbrennt, hat Frankreich das Recht für sich.“). Der soziale Druck war viel zu groß, so Jean-Jacques Becker, als dass man sich der Forderung nach der gemeinsamen Verteidigung der Heimat hätte entziehen können; das habe aber nicht bedeutet, dass die gewohnten politischen Standpunkte und geistigen Einstellungen aufgegeben worden seien, sie rückten bloß in den Hintergrund.

\footnotetext{
${ }^{1}$ Kenneth E. SILVER, Vers le retour à l'ordre. L'avant-garde parisienne et la première guerre mondiale, Paris, Flammarion, 1991.

${ }^{2}$ Ibid., S. 20-22.
} 


\section{Zurück zur Tradition ?}

Apollinaire, der über seine polnische Mutter russischer Nationalität war, stellte schon am 5. August 1914 den Antrag, in die französische Armee inkorporiert zu werden. Er, der schon seit seiner frühen Jugend in Frankreich lebte und schrieb, hatte schon mehrere Anträge auf Naturalisierung als Franzose gestellt und dachte nun, seine Integrationsbereitschaft durch sein militärisches Engagement zu unterstreichen, ähnlich auch der Schweizer Blaise Cendrars oder der Italiener Canudo. ${ }^{3}$ Apollinaire teilte den allgemeinen patriotischen Enthusiasmus, verurteilte jene, die sich wie Delaunay ins Ausland absetzen, um dem Militärdienst zu entgehen. Gegenüber seinem großen Freund Picasso, der sich als Spanier nicht engagierte und auch nicht engagieren musste, war er indes nachsichtig; Picasso sei physisch zu schwach, um sich etwas anderem als seiner unschätzbaren Arbeit als Künstler zu widmen. ${ }^{4}$

Am 5. November 1914 wurde Apollinaire in Nizza dem 38. ArtillerieRegiment zugeteilt und er sollte nunmehr für vier Jahre den Krieg und die französische Uniform nicht mehr verlassen. Sein Freund Picasso schickte ihm schon im Dezember eine Zeichnung, die Apollinaire als Artillerist mit gezücktem Säbel zeigte mit der Inschrift in großen Buchstaben auf blau-weißrotem Band : „Guillaume de Kostrowitzky/Artilleur/1914“. 5

Picasso habe mit dieser Zeichnung, die an die Épinal-Bilder erinnerte, spontan erkannt, in welche Richtung sich der populäre Kunstgeschmack entwickle, meint dazu Kenneth E. Silver. In der Tat stießen die Épinal-Bilder auf große Resonanz. Épinal erinnerte an das zu rückerobernde Lothringen. Die Épinal-Bilder verstanden sich nicht als hohe Kunst ; aber in ihrer Naivität konnten sie mit dem „Primitivismus“ in Verbindung gebracht werden, der den Künstlern der Jahrhundertwende durchaus sympathisch war. ${ }^{6}$ Der Maler Raoul Dufy, der für das Propagandabüro der Regierung arbeitete, entwarf so eine ganze Reihe von graphischen Blättern im Épinal-Stil, die die Alliierten darstellten, und die starke Verbreitung fanden.

In den Augen von Kenneth E. Silver bedeutet das Épinal-Bild von Apollinaire, das Picasso entwarf, eine Abkehr von der avantgardistischen Formen der Vorkriegszeit und eine Hinwendung $\mathrm{zu}$ traditionelleren Ausdrucksweisen. Diese Wende glaubt er auf diskursiver Ebene auch in Cocteaus Zeitschrift Le Mot festzustellen, der eine gleichzeitig

\footnotetext{
3 Cendrars und Canudo hatten schon am 2. August 1914 einen « Appel aux étrangers vivant en France » veröffentlicht : „,Des étrangers amis de la France qui ont appris à l'aimer et à la chérir comme une seconde patrie, sentent le besoin impérieux de lui offrir le bras“. Zitiert bei Annette BECKER, Apollinaire.Une biographie de guerre 1914-1918, Paris, Tallandier, 2009, S. 46.

4 Ibid., S. 48.

5 Abgebildet in SILVER, Vers le retour de l'ordre, S. 31.

6 Ibid., S. 32.

7 Ibid., S. 33-35.
} 
modernistische und antimodernistische Ästhetik der Ambiguität vertrat oder in André Ozenfants Publikation L'Élan, die die Avantgarde mit nationalistischen Argumenten verteidigte. ${ }^{8}$

Vor allem Picasso sei nun mit seinen Bildern „Le Peintre et son modèle“ (1914), „Portrait de Max Jacob“ (1915) und dem „Portrait d'Ambroise Vollard“ (1915) zur Tradition zurückgekehrt. Er orientierte sich an Géricault, Courbet oder Ingres. Kenneth E. Silver zeigt Picassos Entwicklung vor allem über den Vergleich des kubistischen Bildes „Ma Jolie“ (1912) und dem sehr realistischen „Portrait d'Olga“" von 1917 auf.

Apollinaire schien diesen Schritt seines Freundes zu begrüßen und verstand sein eigenes Schaffen im Einklang mit dem Picassos. „,[Mes travaux] que je fais maintenant concorderait mieux avec tes préoccupations présentes. J'essaie de renouveler le ton poétique, mais dans le rythme classique." ${ }^{9}$

Picasso wird nach Apollinaires Kopfverletzung ein sehr klassisches Porträt seines Freundes in Uniform und den Kriegsorden entwerfen („Portrait de Guillaume Apollinaire en uniforme“" [1918]) ${ }^{10}$ analog zu dem Bild des anderen Maler-Freundes, Canudo, der ebenfalls als Freiwilliger diente („Portrait de Riccioto Canudo en uniforme“ $[1918])^{11}$. Apollinaire wird seinerseits den stark „lateinischen“ Charakter des Kubismus betonen, so in einem Aufsatz im Mercure de France :

\begin{abstract}
Tout le mérite de cette 'création' - si mérite il y a - revient sans que cela puisse être contesté à Pablo Picasso et Georges Braque. Ce sont les véritables 'créateurs' d'une école qui pourrait être qualifiée par conséquent de francoespagnole ou plus simplement de latine. Il est vrai que depuis la guerre elle s'est étendue au point qu'il s'en faudrait de peu qu'on pût l'appeller cosmopolite si sa latinité ne s'était encore une fois affirmée par l'absorption importante qu'elle a faite du futurisme italien. Il est juste par conséquent qu'on appelle latine une école dont les principaux adeptes sont français, espagnols et italiens. De toute façon, elle est née sur le sol français et les artistes qui la composent travaillent à Paris, ce qui fait que le qualificatif de 'parisienne' ne lui irait pas si mal. ${ }^{12}$
\end{abstract}

Kenneth E. Silver erwähnt dann noch, dass Apollinaire wie Gide, Proust und Rodin während des Krieges zu den Abonnenten des neo-royalistischen Blattes L'Action française zählte und dass er nach seiner Verwundung in Briefkontakt mit dem Action française-Intellektuellen Léon Daudet stand ${ }^{13}$ offensichtlich weitere Belege des Rechtsdralls von Apollinaire im Kontext des Krieges.

Ibid., S. 42-49.

${ }^{9}$ Brief vom 4. September 1918, zitiert ibid., S. 124.

${ }^{10}$ Abgebildet ibid., S. 95.

${ }^{11}$ Abgebildet ibid., S. 98.

12 Guillaume APOLLINAIRE, « Lettre au Mercure de France », 22. September 1917 ; zitiert ibid., S. 110 .

${ }_{13}$ Ibid., S. 108. 


\section{Apollinaire im Kontext des literarischen Feldes}

2001 veröffentlichte Anna Boschetti, die zuvor, von Bourdieus Feldtheorie ausgehend, Sartre und die Temps Modernes ${ }^{14}$ analysiert hatte, eine Monographie zu Apollinaire unter dem Titel La poésie partout. Apollinaire, homme-époque (1898-1918). Die Autorin ist mit der These Silvers, der eines 'retour à l'ordre' der Avantgarde, und vor allem Apollinaires, während der Kriegsjahre, nicht einverstanden. Sie folgt generell dem Gedanken Bourdieus, dass die soziokulturelle Disposition deklassierter Bürger- oder Adelssöhne oder -töchter, oder Angehörige von Minderheiten zur literarischen Innovation führe. ${ }^{15}$ So zählten in der Tat zahlreiche Ausländer oder Angehörige der jüdischen Minderheit der Gruppe der Symbolisten. Die politische Avantgarde des 20. Jahrhunderts wies dieselben Charakteristika auf : Apollinaire, Cendrars, Marinetti waren Nicht-Franzosen in Paris, Max Jacob war Jude. Diese wagten sich in den riskanten Bereich der avantgardistischen Poesie vor, weil ihnen der "sens du placement" der Einheimischen abging, die sich der weniger riskanten Gattung des Romans zuwandten, so Gide oder die Unanimisten. Apollinaire, der bis zu seinem siebten Lebensjahr in Italien lebte, um dann ab 1899 in den avantgardistischen Kreisen von Paris zu verkehren, zog indes Profit aus seiner sozialen Exzentrizität : er hatte nichts zu verlieren.

Wenn er in der ersten Periode (1901-1906) zunächst sich in eine bestehende Position des literarischen Feldes, dem Prinzip der Einfachheit des „Naturisme“ mit seinen „Rhénanes“ einschrieb, so versuchte er dann diese Position durch eine neue antinaturalistische Ästhetik (1907-1908) zu überwinden, die die Autonomie der Kunst und der Bedeutung der formalen Elemente und der Diskontinuität unterstreicht, so in seinem „Portrait de Matisse“ (1907). Wenn er schon 1908 mit den kubistischen Versuchen von Picasso und Braque vertraut wird, so sucht er in der Poesie ein sprachliches Äquivalent dazu zu schaffen. ${ }^{16}$ Er, der über gute Kenntnisse der Maltechnik verfügt, stellt Picasso andere Maler (Derain, Matisse, Braque, De Chirico, Rousseau) sowie Max Jacob vor, die sich als Gruppe des Bateau-Lavoir konstituieren.

\footnotetext{
${ }^{14}$ Anna Boschetti, Sartre et les 'Temps modernes'. Une entreprise intellectuelle, Paris, Éditions de Minuit, 1985; siehe dazu Joseph JuRT, Das literarische Feld. Das Konzept Pierre Bourdieus in Theorie und Praxis, Darmstadt, Wissenschaftliche Buchgesellschaft, 1995, S. 283295.

„15es écrivains les plus novateurs sont très souvent] des bourgeois dévoyés ou déclassés qui possèdent toutes les propriétés des dominants moins une, parents pauvres des grandes dynasties bourgeoises, aristocrates ruinés ou en déclin, étrangers ou membres de minorités stigmatisées comme les juifs." (Pierre BouRdieu, Les Règles de l'art. Genèse et structure du champ littéraire, Paris, Seuil, 1992, S. 88).

${ }^{16}$ Apollinaire wird aber die Bezeichnung seines Schaffens (wie desjenigen von Max Jacob und Cendrars) durch das von Frédéric Lefèvre vorgeschlagene Label „cubisme littéraire“ ablehnen, weil das suggerieren würde, ihre Poesie sei bloß eine Imitation der kubistischen Malerei.
} 
In den Jahren 1909-1911 findet Apollinaire seinen eigenen Weg, indem er sich von den beiden Gruppen des Unanimisme (um Jules Romains) und der 1909 gegründeten N.R.F. abgrenzt, zwei Gruppen, die im Übrigen ausschließlich aus Franzosen bestehen. 1912/13 erreicht er die herausragende Position einer Avantgarde der Modernität, die die zeitgenössische Welt in die Kunst integriert : „L'œuvre d'un artiste ne doit avoir de rapport qu'avec l'époque où cet artiste vit““. ${ }^{17}$ In enger Zusammenarbeit mit den Malern entwirft er eine Kunst, die Gegenstände des Alltags - Reklame, Zeitungsausdrucke - in die Werke integriert, die der kubistischen Ästhetik der Auflösung und der Rekonstruktion entspricht. Der Kubismus versucht nach Apollinaire die simultane Wahrnehmungsweise des modernen Menschen zu übersetzen. Er wird mit seinem Werk Peintres cubistes. Méditations esthétiques (1913) zum Verteidiger der Innovation auf allen Ebenen. Bezeichnenderweise findet sich auf dem Titelblatt seines Gedichtbandes Alcools (1913) ein von Picasso entworfenes kubistisches Portrait von ihm.

Apollinaire findet Resonanz auch außerhalb Frankreichs, etwas bei Ezra Pound und tritt in Kontakt mit den Berliner Expressionisten der Gruppe Der Sturm sowie mit italienischen Künstlern wie De Chirico und Savinio. Sein Gedicht „Les Fenêtres“ versteht sich wie ein kubistisches Bild mit fragmentierten Inhalten, die nicht mehr über ein lyrisches Ich oder eine metaphorische Kontinuität organisiert werden; es wird nicht mehr zwischen einem Innen und Außen unterschieden. ${ }^{18}$ Das Gedicht entspricht das Delaunays „Fenêtres“, die über Farbkonstruktionen gegen das NichtFigurative tendieren. Einen Schritt weiter geht dann Apollinaire mit der visuellen Poesie, seinen Calligrammes, die die Linearität der Literatur aufzuheben versuchen in Richtung einer nicht hierarchisierten Simultaneität, die ähnlich wie Delaunays „Tour Eiffel“, dem Autor keine Zentralposition mehr zuschreiben.

Hinsichtlich Apollinaires Texten, die er während der vier Kriegsjahre schrieb, kommen nach Anna Boschetti die Interpreten indes sehr schnell zu moralischen (positiven oder negativen) Urteilen und verkennen dabei, dass kollektive Ereignisse wie ein Krieg die gesamte Gesellschaft beeinflussten und so auch die Autonomie des Feldes der kulturellen Produktion in Frage stellten. ${ }^{19}$

Apollinaire habe sich als Freiwilliger engagiert, weil er den Krieg (auf Frankreichs Seite) als legitim einstufte. In seinen Texten bringe er bisweilen einen germanophoben Patriotismus zum Ausdruck, der ihm die Überlegenheit der französischen, lateinischen Zivilisation über die deutsche Barbarei hervorheben ließ. Die Schrecken der Front habe er zu überwinden versucht, indem er die Selbstbeherrschung und die Erkenntnis des Lebens betonte, die

\footnotetext{
${ }_{18}^{17}$ Zitiert ibid., S. 134.

18 Siehe dazu Laurent Jenny, La Fin de l'intériorité. Théorie de l'expression et invention esthétique dans les avant-gardes françaises, Paris, P.U.F., 2002.

19 Anna Boschetti, La Poésie partout, Paris, Seuil, S. 192-193.
} 
ihm diese Erfahrung vermittelten; dabei folgte er einer Art „Religion der Ehre“. Selbst wenn er die positiven Seiten des Militärlebens besang, so sei er auch hin- und hergerissen gewesen zwischen der patriotischen Loyalität und dem Gefühl der Revolte gegen die „patries jalouses“. In dem Gedicht „Allons plus vite“ von 1917 brachte er seine Wut gegen die „Patrons“, die vom Krieg ökonomisch profitierten, zum Ausdruck. ${ }^{20}$

Vor allem der Vers „Ah Dieu ! que la guerre est jolie“ aus dem Gedicht „L'Adieu du Cavalier“ von 1915 wurde Apollinaire vorgeworfen so wie der Gedichttitel „Merveille de la guerre“. Das sind aber nach Anna Boschetti keine Lobpreisungen des Krieges ; die Verse betonten vielmehr das Paradox einer schrecklichen Situation, die ästhetische Emotionen hervorrufen könne. ${ }^{21}$ Seine Haltung entspreche im Übrigen der Orientierung der meisten Vertreter einer „reinen“ Literatur während der Kriegsjahre. Die Ablehnung des Krieges sei nur von ganz wenigen Schriftstellern wie Romain Rolland vertreten worden.

Anna Boschetti sieht bei Apollinaire auch persönliche Prädispositionen für sein Engagement. Er glaubte, Sohn eines Offiziers zu sein, mit dem er sich so zu identifizieren zu können hoffte. „Mourir comme mes aïeux qui étaient tous des guerriers", so definierte er sein Schicksal in einem Brief an seine Geliebte Lou. ${ }^{22}$ Die Experimentierlust scheine in seinen Kriegsgedichten etwas erlahmt zu sein ; aber man finde auch jetzt Versuche, die Simultaneität der Wahrnehmungen zu übersetzen. Die Person des Dichters steht mehr im Vordergrund, so etwa im Gedicht „Merveille de la guerre“: „Je lègue à l'avenir l'histoire de Guillaume Apollinaire/ Qui fut à la guerre et sut être partout. ${ }^{23}$

Der Dichter schreibt sich und der Dichtung nicht mehr bloß eine literarische, sondern auch eine patriotische, politische Funktion zu, was nach Anna Boschetti einherging mit einer Schwächung der poetischen Qualität. ${ }^{24}$ Apollinaire sah sich indessen durch sein militärisches Engagement legitimiert und konnte seine Versuche, innovative ikonische Texte herzustellen weiterverfolgen, während die Künstler in Paris angesichts der Attacken gegen die Avantgarde ihre Ernsthaftigkeit und ihren Patriotismus unter Beweis stellen mussten. ${ }^{25}$

${ }^{20}$ Ibid., S. 193-194.

${ }^{21}$ Ibid., S. 195.

${ }^{22}$ Zitiert ibid., S. 197.

${ }^{23}$ Zitiert ibid., S. 201.

${ }^{24}$ Ibid., S. 202.

${ }^{25}$ Siehe ibid., S. 316 : „Les artistes qui ont participé au combat n’ont pas ressenti, par ailleurs à la différence de ceux qui sont restés à Paris, l'effet culpabilisant des attaques que la guerre a déclenchées contre les innovations des avant-gardes : leur engagement les exemptait de prouver leur patriotisme. C'est ainsi qu'Apollinaire peut continuer à revendiquer la plus totale liberté dans ses expériences : il n'abandonne jamais ses recherches 'calligrammatiques', qui touchent l'aspect iconique et spatial du texte; et il en vient à concevoir une notion de la poésie encore plus ample, qui va au-delà du domaine du langage verbal. Le recours à des moyens pris à d'autres arts (théâtre, danse, musique, cinéma) lui apparaît comme une voie qui ouvre à la 
Als Apollinaire nach seiner Verwundung 1916 nach Paris zurückkommt, ist er enttäuscht über die Indifferenz der Zivilgesellschaft gegenüber den Opfern des blutigen Konfliktes. In diesem Kontext näherte er sich den nationalistischen Schriftstellern, die diese Frage aufgriffen und er beginnt, die Action française zu lesen. Am 20. November 1916 veröffentlicht dieses Blatt einen Leserbrief, in dem Apollinaire Maurras dafür lobt, für die Soldaten aktiv geworden zu sein. Doch nach Anna Boschetti handelt es sich hier nur um eine sehr punktuelle Übereinstimmung. Andere Texte aus dieser Zeit belegten, dass Apollinaire der anarchistisch-aristokratischen libertären Haltung seiner Jugend treu geblieben sei; er verachtet jedes Regime, das die Dichter verkennt.

1916 ist Apollinaire zur führenden literarischen Persönlichkeit seiner Generation geworden und er findet breite Anerkennung auch außerhalb Frankreichs; aber Vertreter der jüngeren Generation, wie die künftigen Surrealisten beginnen sich vom Meister abzusetzen, gemäß einer etablierten Distinktionslogik. Wenn nun der Kubismus in der Öffentlichkeit als subversiv und destruktiv angegriffen wird, so sucht Apollinaire in seinen Briefen an Maurras und Léon Daudet die Legitimität der avantgardistischen Versuche zu verteidigen. In seinem Vorwort zur Ausstellung Matisse-Picasso unterstreicht Apollinaire, dass Picasso nicht der Gefangene eines einzigen Stils sei, sondern sich in unerwartete Richtungen entwickelt habe. Man könne, so Anna Boschetti, die Wendungen und das Oszillieren einer künstlerischen Generation nicht allein als Reaktion auf die Attacken gegen die Avantgarde während des Krieges zurückführen. Die Künstler seien sich bewusst geworden, dass die permanente formale Revolution in eine Sackgasse münden könne, vor allem auch weil das Publikum oft den eingeschlagenen Weg nicht folgen wollte. ${ }^{26}$ Den Willen zur Innovation gab Apollinaire aber nicht auf : „Nous irons plus loin sans avancer jamais“, schreibt er so in seinem Gedicht „Toujours“.

poésie des possibilités nouvelles. En effet, ses tentatives 'multimédias' avant la lettre annoncent une des directions les plus caractéristiques et les plus fécondes de la recherche artistique du $\mathrm{XX}^{\mathrm{e}}$ siècle."

${ }^{26}$ Ibid., S. 230-231: „Si les novateurs sont sensibles aux accusations des fauteurs de l'ordre ancien, c'est qu'ils sont conscients des impasses qu'implique la logique de la révolution permanente : le vieillissement accéléré des inventions, la difficulté toujours croissante de trouver des voies nouvelles, le sentiment d'avoir épuisé toutes les possibilités, la mise en cause radicale de l'art et de sa fonction, l'écart qui s'est creusé entre les recherches de l'avant-garde et les goûts du public cultivé. Ce sentiment d'impasse est présent chez Apollinaire dès avant la guerre, comme en témoigne le dialogue sur l'art et la poésie entre le protagoniste du 'Poète assassiné' et le personnage inspiré de Picasso. [...] Dans un champ autonome, les transformations des modèles artistiques sont indépendantes, à l'origine, des événements externes, bien que ceux-ci exercent des effets, car ils peuvent favoriser certaines positions plus que d'autres." 


\section{Apollinaire - Zeuge einer 'Kriegskultur'}

2009 veröffentlichte dann die Historikerin Annette Becker eine Monographie unter dem Titel Guillaume Apollinaire. Une biographie de guerre 1914-191827. Die Autorin ist eine eigentliche Spezialistin des Ersten Weltkrieges. Sie beruft sich auf den Ansatz der „culture de guerre“ und betrachtet den Krieg nicht bloß in seiner militärischen und politischen Dimension, sondern als gesellschaftliches Gesamtphänomen, in das alle Bereiche der Gesellschaft involviert sind. ${ }^{28}$ Sie bezieht sich nicht nur auf die literarischen Werke von Apollinaire, sondern auf das Gesamt seiner Verlautbarungen, vor allem auch auf seine umfangreiche Korrespondenz, seine Zeichnungen, Gedichte, seine journalistischen Texte. Die Haltung Apollinaires wird so in ein globales Panorama eingebunden, ohne dass dabei seine Singularität vernachlässigt würde ; seine gesamten Äußerungen stellen, so die Autorin, ein außergewöhnliches Zeugnis dar, das erlaube, den großen Krieg besser zu verstehen. Diese Einbettung ermöglicht aber auch, Apollinaire besser einzuschätzen.

Die Autorin rekonstruiert zunächst die Zeugnisse eines enthusiastischen und bewusst naiven Patriotismus der ersten Augustwochen, aber auch das Gefühl der Einsamkeit des Dichters, da die meisten Freunde zum Militärdienst aufgeboten wurden; wie die meisten denkt er an einen kurzen und glorreichen Krieg. Wenn er dann als 2e canonnier-conducteur des 38. Artillerie-Regiments in Nîmes ab November 1914 ausgebildet wird, so spürt er hier die physische und symbolische Gewalt, die ihn verändert. Wenn er sich im April 1915 für den Einsatz an der Front meldet, dann auch in der Hoffnung auf einen militärischen Aufstieg und er bereitet sich mit einer gewissen Begeisterung auf die technische Prüfung vor. Seine Militär- und Kriegserfahrungen teilt er vor allem in Briefen an 'seine' Frauen mit : Lou Louise de Coligny-Châtillon - und Madeleine Pagès, die junge Frau, die er im Zug im Januar 1915 kennen gelernt hatte. Die Stillage seiner Briefe und der beigelegten Gedichte wird so auch durch die jeweiligen Empfänger(innen) bestimmt ; in den Briefen an die Frauen stellt er seine Mannhaftigkeit in den Vordergrund und spart auch nicht mit erotischen Anspielungen und humorvollen Wortspielen. Die Briefe an Fernand Fleuret und Francis Picabia zeugen indes vom Ernst, ja von der Tragik des Krieges. Widersprüchliche Gefühle kommen zum Ausdruck, „,consentement à la guerre; haine de la guerre“. ${ }^{29}$ Annette Becker stellt die vielen Oxymora in seinen Äußerungen fest, die das Oxymoron der Kriegserfahrung zum

\footnotetext{
27 Annette BECKER, Guillaume Apollinaire. Une biographie de guerre, 1914-1918, Paris, Tallandier, 2009.

${ }^{28}$ Den Ansatz teilt sie mit Jean-Jacques Becker, Stéphane Audoin-Rouzeau sowie Gerd Krumeich.

${ }^{29}$ Ibid., S. 62.
} 
Ausdruck bringen : „L'écrivain [...] fait de sa vie au front l'oxymore des oxymores, entre tragique et comique, danger et calme. ${ }^{630}$

Die ideologische Rechtfertigung des Krieges bei Apollinaire ist nun keineswegs originell. Vom Feind spricht er nur als den 'boches', keine Spur einer Solidarität mit den Soldaten jenseits der Front. Deutschland wird kulturelle Inferiorität zugeschrieben gemäß dem gängigen Barbaren-Topos („,e pays d'idiotie et de médiocrité") und Apollinaire bedauert seine Kontakte mit deutschen Künstlern vor dem Krieg. ${ }^{31}$ Frankreich und die lateinischen Länder sind indes der Hort der Zivilisation: „L'innocence du pays que je défends me donne plus d'espoir que tout au monde. ${ }^{\text {‘32 }}$

1915 publiziert Apollinaire mit Hilfe der Druckmaschine und des Papiers der Schützengrabenpresse eine kleine Gedichtsammlung unter dem Titel $L a$ Case d'Armons - in einer Auflage von 25 Exemplaren ; der Titelbegriff bezeichnet eine Nische für persönliche Effekten im Artillerie-Geschütz; es handelt sich bei dieser kleinen Gedicht-Sammlung zumeist um Calligramme, die einer avantgardistischen Schreibweise entsprechen: ,[C]'est le comble de l'avant-gardisme ${ }^{\text {‘33 }}$ schreibt Annette Becker: „D'un point de vue littéraire il n'abandonne en rien la forme nouvelle qu'il avait expérimentée en 1914.“34 Die modernste Schreibweise ging hier einher mit der artisanalsten Herstellungsform auf einer Soldatendruckpresse. Gleichzeitig sendet er ein Exemplar seines Bändchens an seine neue Geliebte Madeleine als „fixation d'un moment de ma vie“. ${ }^{35}$ Seine Briefe und seine Gedichte halten so permanent die Verbindung zwischen der militärischen Front und der Heimatfront aufrecht (Annette Becker betont von ihrem Ansatz der „Kriegskultur“ die Verbindung der beiden „Fronten“: sie spricht dann von „front domestique“ und nicht vom „arrière“). Alle Äußerungen Apolllinaires sind so „le journal de tranchée individuel d'un combattant qui, comme les autres, se débat avec l'éloignement et la souffrance, les rumeurs et les réalités, la découverte de l'ennemi, de ses camarades de tranchée, du feu qui tue."'36 Letztlich aber, so schreibt er im Dezember 1915, kann kein Schriftsteller den schlichten Schrecken und das geheimnisvolle Leben des Schützengrabens beschreiben.

An der Front wirkte er als Verbindungsmann zu Pferd und nimmt aus dieser Perspektive den Krieg akustisch wahr, wie „barocke Musik““. ${ }^{37}$ In

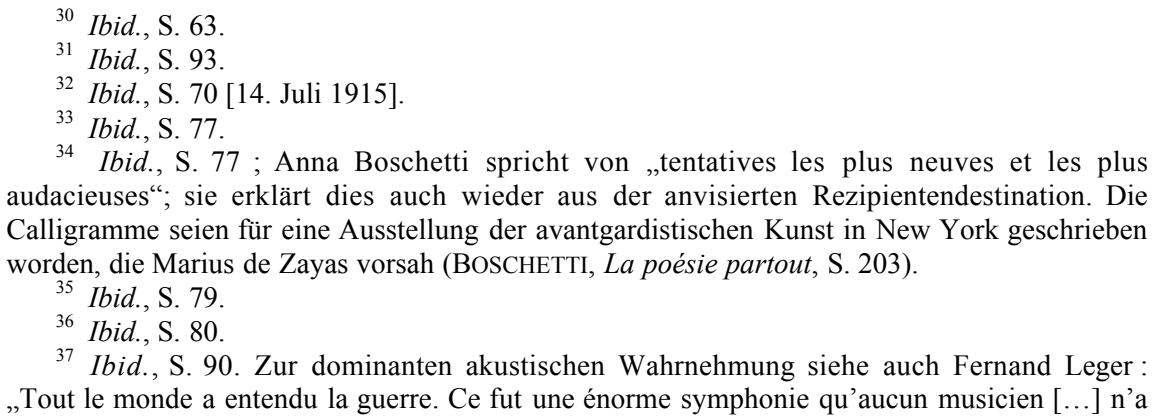

${ }^{37}$ Ibid., S. 90. Zur dominanten akustischen Wahrnehmung siehe auch Fernand Leger: „Tout le monde a entendu la guerre. Ce fut une énorme symphonie qu'aucun musicien [...] n'a 
diesem Kontext ist auch das kleine Gedicht „L'adieu du cavalier“ vom September 1915 zu situieren :

L'Adieu du Cavalier

Ah Dieu ! que la guerre est jolie

Avec ses chants ses longs loisirs

La bague si pâle et polie

Et le cortège des plaisirs

Adieu ! Voici le boute-selle !

Il disparut dans un tournant

Et mourut là-bas, tandis qu'elle

Cueillait des fleurs en se damnant. ${ }^{38}$

Apollinaire sandte dieses Gedicht an Madeleine, die Adressatin ist hier nicht unwichtig ; gleichzeitig unterstrich er, dass das Gedicht Teil eines „petit roman poétique guerrier" sein sollte. Die Serie mit diesen Gedichten unter dem Titel „Le médaillon toujours fermé“ sandte er an seine Ex-, die Malerin Marie Laurencin, damit sie diese zugunsten einer karikativen Organisation illustriere : „Apollinaire fait au moins d'une pierre trois coups, amoureux, charitable, littéraire". ${ }^{39}$

Das skandalöse Oxymoron „Ah Dieu ! que la guerre est jolie“ wurde in den Augen von Annette Becker nach dem Tod von Apollinaire zum „vers le plus célèbre et le plus incompromis“ “. ${ }^{40} \mathrm{Nach}$ ihr entsprechen diese Verse den Erfahrungen vieler Schriftsteller und Intellektuellen während des Krieges. Mit der Erinnerung an den Krieg verbanden sie die Erfahrungen von Schönheit und Gewalt, von Verzweiflung und Faszination. Der Maler André Masson, der sich als Freiwilliger für den Kriegsdienst gemeldet hatte und 1917 schwer verwundet wurde, wusste sich eins mit der Übersetzung der Kriegserfahrung von Apollinaire ; er kam explizit auf den berühmten Vers des Dichters zurück. Auch für ihn war die Gewalt ein Teil des Lebens im Krieg und man musste sich darauf einstellen. Wenn der Krieg nur permanenter Horror gewesen wäre, wie es Barbusse in Le Feu oder Remarque in Im Westen nichts Neues es darstellen, dann wäre das nicht auszuhalten gewesen. Es gab aber auch Kompensationen, so Masson. Es gab auch veritable Glücksmomente : Manchmal gab es Dinge zu sehen, die schön waren und wären es nur die Leuchtfeuer am Abend. Die Geschosse, der Geruch des Schlachtfeldes konnten berauschen. All' das hat Apollinaire gesehen. Nur ein Dichter konnte

encore égalée.“ (Zitiert ibid., S. 130.) Vgl. Jean EchenOZ, 14, Paris, Éditions de Minuit, 2012, S. 79 : „Tout cela ayant été décrit mille fois, peut-être n'est-il pas la peine de s'attarder encore sur cet opéra sordide et puant. Peut-être n'est-il d'ailleurs pas bien utile non plus, ni très pertinent, de comparer la guerre à un opéra, d'autant moins quand on n'aime pas tellement l'opéra, même si comme lui c'est grandiose, emphatique, excessif, plein de longueurs pénibles, comme lui cela fait beaucoup de bruit et souvent, à la longue, c'est assez ennuyeux."

${ }^{38}$ Zitiert ibid., S. 95.

${ }^{39}$ Ibid., S. 95.

${ }^{40}$ Ibid., S. 95-96. 
dies zum Ausdruck bringen. Er hat nicht eine Apologie des Krieges vorgelegt, sondern schlicht die Apologie des Lebens im Tod, des Friedens im Krieg ${ }^{41}$ : „Pour lutter contre la déshumanisation de la grande tuerie, l'écrivain combattant en vient à humaniser tout ce qui l'entoure“. ${ }^{42}$

Er teilt das Leben der Soldaten; kommt auf religiöse Praktiken zurück, sammelt Kirchenfenstersplitter der Kathedrale von Reims und bezieht sich auf Jeanne d'Arc. Er beobachtet, wie Soldaten kleine Kunstgegenstände aus Trümmermaterial herstellen, um die Zeit totzuschlagen - das Kunsthandwerk der Schützengräben als Kontrast zum industrialisierten Krieg.

Apollinaire meldet sich dann zur Infanterie, wo er im November 1915 den Grad eines Unteroffiziers erhält. In den Grabenkämpfen erfährt er noch viel unmittelbarer die Schrecken des Krieges: „Je sens maintenant toute l'horreur de cette guerre [...]. Les souffrances de l'infanterie sont au-dessus de tout ce qu'on peut imaginer $[\ldots]^{“{ }^{4} .}{ }^{43}$ In dieser Zeit schreibt er seine eindrücklichsten Gedichte zu den Schrecken der Front. Er vergleicht die Schützengräben mit einem Vampir, der alles verschlingt. Das Gedicht „Ombre“ erscheint als ein Schrei in tiefster Not. Annette Becker konstatiert bei Apollinaire in den letzten Monaten an der Front ein eigentliches Kriegstrauma. „Je déteste cette guerre", schreibt er nun im Dezember 1915. Im März 1916 wird er, drei Tage nachdem ihm das französische Bürgerrecht verliehen wurde, von einem Granatsplitter an der Schläfe in der Nähe des Chemin des Dames schwer verletzt und muss mehrmals operiert werden (Schädelbohrung). Er wird mit der Tapferkeitsmedaille ausgezeichnet. Nach mehreren Krankenhausaufenthalten kann er zurück nach Paris, bleibt aber mobilisiert, arbeitet in der Zensurabteilung des Kriegsministeriums und im Kolonialministerium. Er behält seine Uniform und Photos und Bilder halten ihn mit seinem verbundenen Kopf fest.

Er nimmt nun seine intellektuelle Aktivitität wieder auf, beendet die Gedichtsammlung Calligrammes, den Erzählband Le Poète assassiné, schreibt das surrealistische Stück Les mamelles de Tirésias. Gleichzeitig trauert er der Zeit an der Front trotz aller Schrecknisse nach. Zwei Tage vor Friedensschluss, am 9. November 1918, erliegt er mit 38 Jahren dem, was man damals „Spanische Grippe“ nannte, was aber nach Annette Becker eine Vogelgrippe war, die nicht aus Spanien, sondern aus China über die USA eingeschleppt wurde und die allein in Frankreich 240.000 Tote forderte. Blaise Cendrars begleitete Apollinaire in seinen letzten Stunden und bei der Beerdigung auf dem Père Lachaise. In der Zeitschrift SIC gedachte er des Verstorbenen :

Amis

Apollinaire n'est pas mort.

Vous avez suivi un corbillard vide

${ }^{41}$ Entretiens avec André Masson [1985] zitiert ibid., S. 96.

${ }^{42}$ Ibid., S. 99.

43 Brief vom 16. Dezember 1915, zitiert ibid., S. 145. 


$$
\begin{aligned}
& {[\ldots]} \\
& \text { Et voilà que se lève une nouvelle génération } \\
& {[\ldots]} \\
& \text { Et ils parlent tous la langue d'Apollinaire. }
\end{aligned}
$$

Nach der Lektüre des Buches von Annette Becker würde ich in Bezug auf Apollinaire nicht mehr von einer ästhetischen Valorisierung des Krieges sprechen, sondern eher von einer Humanisierung der paradoxen Realität des Krieges durch vielfältige innovative literarische Formen in einem eindrücklichen, sehr persönlichen Zeugnis, das gleichzeitig für zahllose andere zeugt ${ }^{45}$ - oder um es mit den Worten von Annette Becker zu sagen : „Apollinaire avait recherché du sens dans le désastre de la guerre, sens trouvé dans les paradoxes renouvelés du conflit et des représentations qu'il en avait tirées, de la guerre désirée pour atteindre la paix, de la civilisation brisée pour mieux la ressaisir"“. ${ }^{46}$

\footnotetext{
${ }^{44}$ Blaise CENDRARS, „Hommage à Guillaume Apollinaire“, SIC, Januar 1919, zitiert ibid., S. 218.

${ }^{45}$ Annette Becker spricht ihrerseits in Bezug auf die Einschätzung Apollinaires von einer Debatte « qui oppose depuis quatre-vingt-dix ans, ceux qui n'ont vu que les héros et ont tu les souffrances à ceux qui n'ont vu que les victimes et on tu le consentement », ibid., S. 255.

${ }^{46}$ Ibid., S. 234-235.
} 